\title{
El aluvión del 9 agosto 2015 en Alto Patache, región de Tarapacá, Desierto de Atacama'
}

\author{
Héctor Orellana², Juan-Luis García ${ }^{3}$, Carla Ramírez ${ }^{4}$ \\ y Nicolás Zanetta ${ }^{5}$
}

\begin{abstract}
RESUMEN
Durante el 9 agosto de 2015 (9A) (año El Niño Fuerte) se produjeron lluvias torrenciales anómalas que favorecieron el desarrollo de aluviones en el Norte Grande de Chile. En la Estación Atacama UC de Alto Patache, cordillera de la Costa de la región de Tarapacá, se registraron 51,6 $\mathrm{mm}$ en 6 horas, condición que superó rápidamente el umbral de infiltración del suelo gatillando aluviones en las quebradas aledañas. En la cuenca de control de 7,2 Ha se cuantificó una erosión máxima de $1.070 \mathrm{~m}^{3}$ de sedimentos, parte de los cuales se depositaron en un cono aluvial de $\sim 637 \mathrm{~m}^{3}$ de material. En la sección inferior de la cuenca se estimó caudales medios de $11 \mathrm{~m}^{3} \mathrm{~s}^{-1}$. La secuencia del 9A es diferenciada en tres fases: Inicio, Peak y Término que incluyen la dinámica meteorológica e hidrogeomorfológica conjunta. El aluvión 9A descrito en este estudio tiene precedentes en Alto Patache, donde periodos de relativa estabilidad geomorfológica son interrumpidos por Iluvias intensas con alto impacto en el paisaje en respuesta al fenómeno ENSO.
\end{abstract}

Palabras claves: Aluvión 9 agosto 2015, Estación Atacama UC Oasis Niebla Alto Patache, morfoestratigrafía, El Niño Oscilación del Sur (ENOS).

\begin{abstract}
During the 09 August 2015 (strong El Niño year) heavy rains fell in the coastal Atacama Desert, including our Research Station Atacama UC_Alto Patache (cordillera de la Costa, Tarapacá Region). In this study we provide a detailed analysis of the meteorology and hydrogeomorphic response to the $51,6 \mathrm{~mm}$ in 6 hours of rain recorded in Alto Patache. In our control basin $\left(7.2 \mathrm{Ha}\right.$ ) we estimated a maximum erosion of $\sim 1070 \mathrm{~m}^{3}$, which were partially stored in the distal alluvial fan, where we quantified $637 \mathrm{~m}^{3}$ of material. We estimated a mean water discharge of $11 \mathrm{~m}^{3} \mathrm{~s}^{-1}$ at the lower basin. The comparison between the meteorological and morphostratigraphical data allows us to define three phases that best characterize the anatomy of the 9A: Initial, Peak and End. We conclude that Alto Patache embraces an overall geomorphic stability that is interrupted recurrently by debris flows linkable to ENSO variability.
\end{abstract}

Key words: Debris flow 9 August 2015, Estación Atacama UC_Oasis Niebla Alto Patache, morphostratigraphy, ENSO.

Artículo recibido el 10 de mayo de 2017, aceptado el 25 de agosto de 2017 y corregido el 17 de octubre de 2017. 
La hiperaridez reinante en el desierto de Atacama permite una excelente preservación morfológica y estratigráfica de los procesos geológicos en el tiempo (Dunai et al., 2005; Quezada et al., 2010) Esta condición le ha entregado al Atacama una situación de estabilidad morfogenética única en nuestro planeta (Hartley et al., 2005). Por esto, solo en raras ocasiones se ha podido dimensionar el efecto de procesos geológicos y geomorfológicos en el paisaje. Este aspecto ha imposibilitado un entendimiento completo de la dinámica fisiográfica del desierto extremo y sus causas. Siendo uno de estos procesos los aluviones, que lo entendemos como tipo de movimiento brusco (súbito) de tierra mezclado con agua, que se asocian generalmente a flujo de detritos y de barro (Hauser, 2000). Es sabido que las ocurrencias de estos fenómenos son desencadenadas por eventos de pluviosidad extraordinarios y anómalos, dado que las precipitaciones en el Norte Grande de Chile son prácticamente casi nulas (Ortlieb y Vargas, 2003; Vargas et al., 2006). No obstante, bajo condiciones océano -atmosféricas del fenómeno de El Niño puede precipitar en dicha zona, generando, cuando la duración e intensidad del evento es suficiente, activación de quebradas y aluviones (Ortlieb, 1995; Vargas et al., 2000; Vargas et al., 2006; Houston, 2006; Ortega, 2014). Los depósitos sedimentarios y el registro estratigráfico de los aluviones representan entonces un registro de alto interés climático (Vargas y Ortlieb, 1998; Ortlieb y Vargas, 2003; Vargas et al., 2000). Este trabajo tiene como objetivo la caracterización morfo-estratigráfica del aluvión del 9 de agosto (9A) del 2015, en la Estación Atacama UC Oasis Niebla Alto Patache (2048'S, $\left.70^{\circ} 09^{\prime} \mathrm{O}\right)$, en la cordillera de la Costa del desierto de Atacama. Ese día una lluvia de alta intensidad gatilló aluviones en sectores de Tocopilla y el sur de Iquique. El estudio busca aportar antecedentes sobre el factor meteorológico, así como la respuesta hidrológica de la cordillera de la Costa a partir de datos obtenidos en una pequeña cuenca en Alto Patache. En adición, en el texto contrasta la estratigrafía asociada al evento 9A con aquella previa con la intención de entender la recurrencia de estos fenómenos aparentemente anómalos en la región. El trabajo provee de datos únicos que responden a la oportunidad de entender la relación de la meteorologia medida in situ con los procesos geomorfológicos del aluvión del $9 \mathrm{~A}$.

\section{Área de Estudio}

Alto Patache ( 800 m s.n.m., Figura $\left.N^{\circ} 1\right)$ está emplazado en la cordillera de la Costa, sobre el gran escarpe costero (Orellana, 2010; Sepúlveda et al., 2014) del norte de Chile, al interior de la Estación Atacama UC Oasis Niebla Alto Patache. El clima en esta zona del Desierto de Atacama es hiperárido (normales de $\mathrm{pp}=\mathrm{c} .0 \mathrm{~mm}$ año-1), aspecto que es solo interrumpido durante lluvias ocasionales y de alta intensidad, normalmente durante fases El Niño (Manrique et al., 2010). El aspecto más característico del clima regional es la frecuencia de días nublados y la presencia cuasi-permanente de neblina o camanchaca (Cereceda et al., 2008a). La neblina proporciona la principal fuente de humedad, que da vida al Oasis de Niebla de Alto Patache (Pinto et al., 2001; Egaña et al., 2004; Cereceda at al., 2008b; Garreaud et al., 2008; Latorre et al., 2011). Este clima está determinado por la convergencia de multi-factores como son su posición latitudinal, que lo ubica en el sector del descenso de la celda convectiva de Hadley; además de la gran elevación de la cordillera de los Andes, que actúa como una barrera orográfica para la humedad proveniente de advección tropical-subtropical del este; así mismo, la influencia del anticiclón de Pacífico Sur y la presencia de la corriente de Humboldt, asociada a la surgencia de agua profundas favoreciendo la generación de una inversión térmica y una estabilidad atmosférica generalizada (Nishiizumi et al., 1998; Garreaud, 2011). La geología de Alto Patache está conformada por rocas andesíticas, pertenecientes a la Formación Oficina Viz, (Jurásico Medio a Superior), sobreyacidas por gravas 
asociadas a la formación de Alto Hospicio (Neógeno), presentes en las quebradas (Orellana 2010; Sepúlveda et al., 2014). Estos sedimentos incluyen secuencias de gravas, arenas y limos de ambiente aluvial en un clima árido o semiárido desarrolladas en el Mioceno (Sepúlveda et al., 2014). La presencia de una aparente falla inversa, asociable al sistema tectónico de formación del gran escarpe costero (Sepúlveda et al., 2014), ha dado lugar a una depresión encerrada por relieves acolinados en Alto Patache, generando desniveles locales de $70 \mathrm{~m}$ y gradientes que pueden superar los $20^{\circ}$. Por su parte, la depresión ha sido rellenada por depósitos aluviales, que se disponen en discordancia angular sobre la Formación Oficina Viz. Estos depósitos en la actualidad pueden incluir una cubierta depósitos eólicos, que sobreyacen a coluvios y aluvios (Orellana, 2010). No obstante, prima la acción morfogenética del viento que ha determinado superficies onduladas, la deflación de los materiales finos y la formación de pavimentos desérticos, así como el relleno de quebradas dando lugar a una topografía suave. La depresión intermontana posee una inclinación hacia el noroeste hacia el escarpe costero.

Figura No 1

Localización de la Estación Atacama UC-Oasis Niebla Alto Patache en la cordillera de la Costa,

Desierto de Atacama

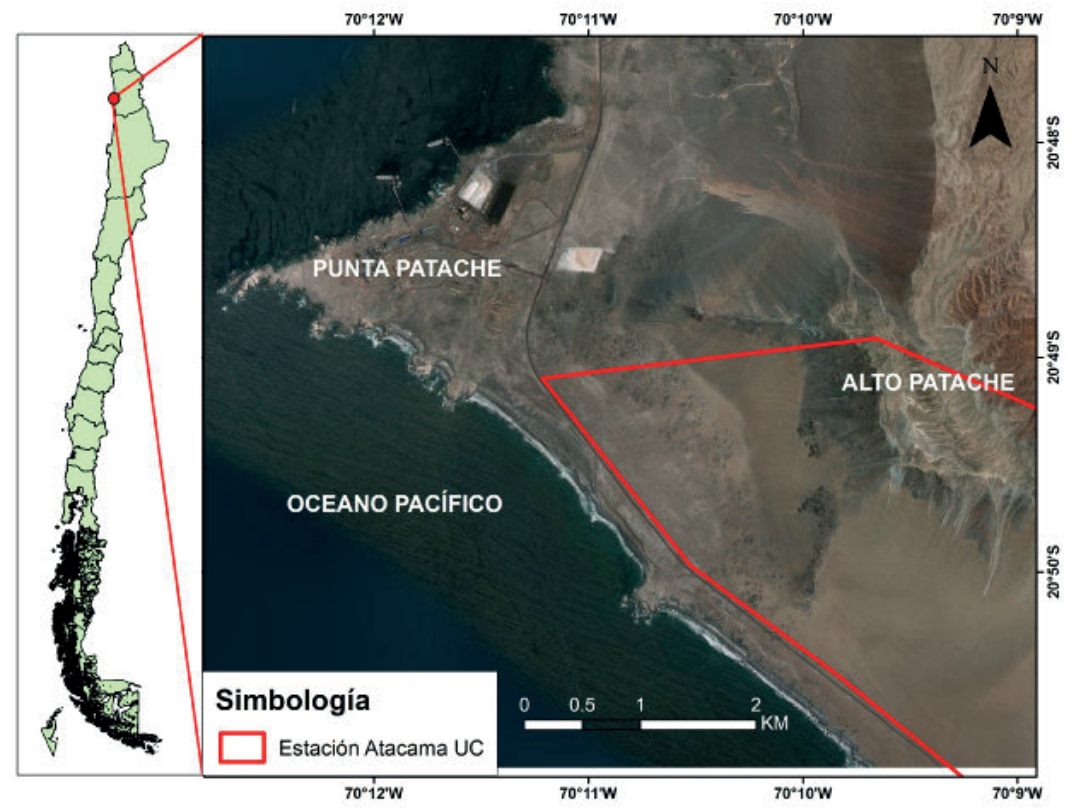

Fuente: Elaboración propia.

\section{Metodología}

Los datos meteorológicos de este estudio se obtuvieron de la estación meteorológica localizada en Alto Patache (850 m s.n.m.), la cual cuenta con sensores específicos para el monitoreo de precipitaciones (Texas Electonics TR525), temperatura y humedad relativa del aire (Campbell Scientific HMP45), presión atmosférica (Vaisala PTB110) y velocidad y dirección del viento (Young 03002). Los datos generados fueron almacenados en un datalogger automático (Campbell Scien- 
tific CR1000), con una resolución temporal de 15 minutos. Esto permitió obtener los registros atmosféricos durante las horas previas al evento, durante el evento y posterior al evento con alta resolución.

La caracterización hidrogeomorfológica, entendida como la respuesta hídrica y geomorfológica, incluyó la determinación de la erosión de arrastre y sedimentación aluvional durante el evento $9 \mathrm{~A}$ en la cuenca control de Alto Patache (Figura No 2). Esta cuenca donde realizamos nuestra investigación posee un área de $72.175 \mathrm{~m}^{2}$ (7,2 Ha), aproximadamente. En esta cuenca se consideraron 26 puntos de monitoreo denominados en orden alfabético desde la $\mathrm{A}$ hasta la Y, distribuidas en las dos quebradas tributarias principales, denominadas quebrada $N^{\circ} 1$ y $N^{\circ} 2$ (Figura $N^{\circ} 3$ ). En ellas se determinó la sección transversal media erosionada durante el $9 \mathrm{~A}$ y la altitud de cada sección, obtenidas por un GPS, modelo Garmin Gpsmap 60csx (error $\pm 3 \mathrm{~m}$ ). Estos puntos están distanciados entre 5 a 25 metros, siguiendo el criterio de representatividad de cada tramo de la quebrada de forma de incluir con el mayor detalle posible la variedad de geometrías encontradas. Por ejemplo, luego de cambios visibles en la sección transversal de las quebradas se estableció un punto de monitoreo. A partir de estos datos y considerando la ecuación de continuidad para flujo permanente, se estimó el caudal descargado durante el 9A, que se obtiene de la multiplicación de área de la sección $\left(\mathrm{m}^{2}\right)$, por la velocidad media $\left(\mathrm{m} \mathrm{s}^{-1}\right)$. El calado del flujo uniforme se calculó mediante la fórmula de resistencia de Manning. Utilizamos este método dada su simplicidad y universalidad para el cálculo de escurrimiento en canales abiertos. Todos los datos fueron levantados en terreno y se utilizó un coeficiente de rugosidad de 0,04 de los parámetros propuestos por Manning, el cual es el de mayor semejanza a las condiciones del área de estudio. De modo de obtener un valor de descarga hídrica se consideró el flujo como newtoniano y permanente (caudal líquido), a pesar de que el flujo incluye un transporte de sedimentos significativo. Por ende, el resultado obtenido es solo una aproximación a la realidad.

Calculo del Caudal

$\mathrm{Q}=\mathrm{V}^{*} \mathrm{~A} \quad(1)$

Donde:

$\mathrm{Q}=$ Caudal ( )

$\mathrm{V}=$ velocidad media ()

$A=$ área del canal $\left(\mathrm{m}^{2}\right)$

Ecuación de resistencia de Manning

$$
V=\frac{1}{n} * R^{\frac{2}{3}} * S^{\frac{1}{2}}
$$

Donde:

$\mathrm{V}=$ velocidad media ()

$\mathrm{n}=$ Coeficiente de rugosidad de Manning.

$\mathrm{R}=$ Radio Hidráulico $(\mathrm{m})=\left(\frac{\text { Área }}{\text { Perímetro }}\right)$

$\mathrm{S}=$ Pendiente media .

$S=\frac{z_{1}-z_{2}}{\sqrt{\left(x_{1}-x_{2}\right)^{2}+\left(y_{1}-y_{2}\right)^{2}}}$ 
Dónde:

$\mathrm{Z}=$ altitud

$X=$ longitud

$Y=$ latitud

Para obtención de las medidas morfométricas de las quebradas y la cuenca de control, se calculó el área $\left(\mathrm{m}^{2}\right)$ y perímetro $(\mathrm{m})$, a través del uso de un sistema de información geográfica (SIG), específicamente el software libre QGIS versión 2.18.0.

Finalmente, para la obtención del volumen de sedimentos depositados en el cono aluvial a la salida de la cuenca de control, utilizamos marcadores de profundidad instalados pre-evento y observaciones de terreno. Previo al 9A, 8 tubos PVC habían sido instalados a una profundidad que se logró definir en terreno por la marca de decoloración a la intemperie. La diferencia entre la superficie actual y la superficie original pre 9A, determinada a partir de los tubos, nos proporcionó una medida absoluta de acumulación de sedimentos en varios puntos del cono aluvial (Figura No 3). Con estos datos obtenidos, se desarrolló una interpolación con kriging, como manera experimental de aproximarnos a un volumen total del depósito aluvial.

Para el caso del análisis estratigráfico, se efectuó la descripción de las facies e interpretación genética de los depósitos reconocidos en el cono aluvial a través de la excavación de calicatas y en los perfiles expuestos de las quebradas estudiadas. Se realizó la diferenciación de litofacies según los criterios estratigráficos de Boggs (2012) y Miall (2016).

Figura No 2

Cuenca de Control, incluyendo las quebradas y cono aluvial estudiados en este trabajo asociados al evento $9 \mathrm{~A}$.

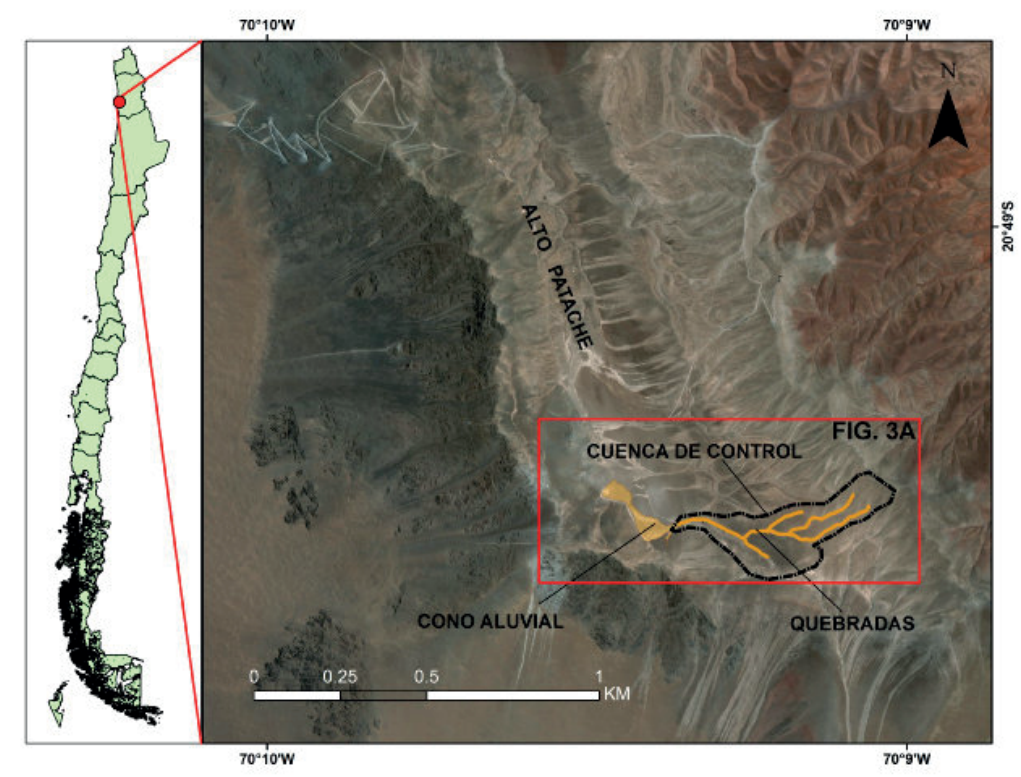

Fuente: Elaboración propia. 
Figura No 3

Puntos de control donde se midió la sección transversal de las quebradas ( $N^{\circ} 1$ y $N^{\circ} 2$ ) y cono aluvial del 9A. A) Los puntos en las quebradas están indicados por las letras A-Y y aquellos en el cono aluvial por las letras $A^{\prime}-E^{\prime}$. B) Medición de la sección transversal de quebradas. C) Medición de la profundidad del cono aluvial a partir de conos PVC instalados pre-9A. Se muestra la parte del tubo que fue enterrada entre la decoloración inferior y la línea negra en la mitad superior.

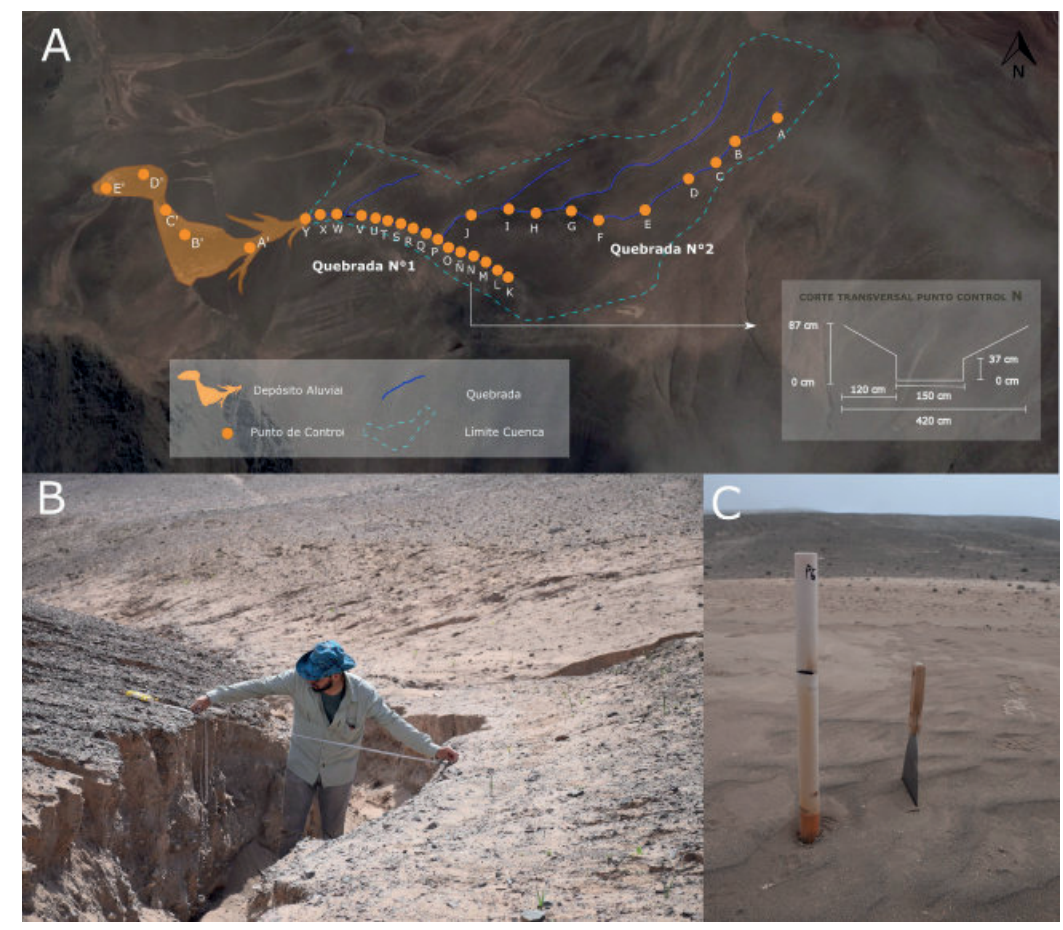

Fuente: Elaboración propia.

\section{Registros atmosféricos y caracterización hidrogeomorfológica del evento 9A}

\section{Meteorología}

Los datos obtenidos en la estación meteorológica de Alto Patache evidencian que el evento de precipitación ocurrió entre las 07:00 y 13:00 horas del día 9 de agosto del 2015. Se registró una precipitación total de 51,6 mm en 6 horas. La anatomía de las lluvias y meteorología general del 9A se presenta en la Figura $N^{\circ} 4$. La secuencia meteorológica indica que entre las 02:00 y 04:00 horas se registró una baja abrupta de $4 \mathrm{hpa}$, indicando el ingreso de un sistema de baja presión. Entre las 03:15 y 05:00 horas, la presión ya se había recuperado, manteniéndose estable hasta las 09:30 horas. La precipitación se inició cerca de las 07:00, con una temperatura de 13,6 $6^{\circ} \mathrm{C}$ y con una dirección de viento preferente $\mathrm{N}$ y NE, cuya velocidad predominante fue entre los rangos 5,7 a 8,8 m.s.-1. Durante los primeros 45 minutos (07:00 - 07:45 horas), se registró un total de 4,8 mm de lluvia, el que fue en aumento hasta los $9,8 \mathrm{~mm}$ hasta las 10 horas, que es cuando se acaba el primer pulso de precipitación. Durante esta primera parte del 9A, la presión atmosférica, la velocidad del viento y la temperatura se elevaron a $920 \mathrm{hpa}, 14,2^{\circ} \mathrm{C}$ y $17,15 \mathrm{~m} \mathrm{~s}^{-1}$, respectivamente. $A$ 
las 10:30 comienza la segunda fase de lluvias en Alto Patache y se mantiene hasta las 13:00 horas, periodo en el que se registraron $41,60 \mathrm{~mm}$ de agua caída. Estas precipitaciones coinciden con la mayor velocidad de viento registrada durante el evento $9 \mathrm{~A}$, que fue de $17,3 \mathrm{~m} \mathrm{~s}^{-1}$, y que ocurrió con un vector de dirección Oeste. La máxima intensidad de lluvia registrada durante esta fase de precipitación, fue de 20,4 mm caídos en 15 minutos entre las 11:15 y las 11:30 horas, en coincidencia con un ascenso marcado de la presión atmosférica. Las horas posteriores al evento, entre las 13 y 15 horas, se caracterizaron por una baja en la intensidad de los vientos (3,6 a 5,7 $\left.\mathrm{m} \mathrm{s}^{-1}\right)$ y un cambio de su dirección a Sur (Figura No 4).

Figura No 4

Condiciones atmosféricas pre, durante y post evento $9 \mathrm{~A}$

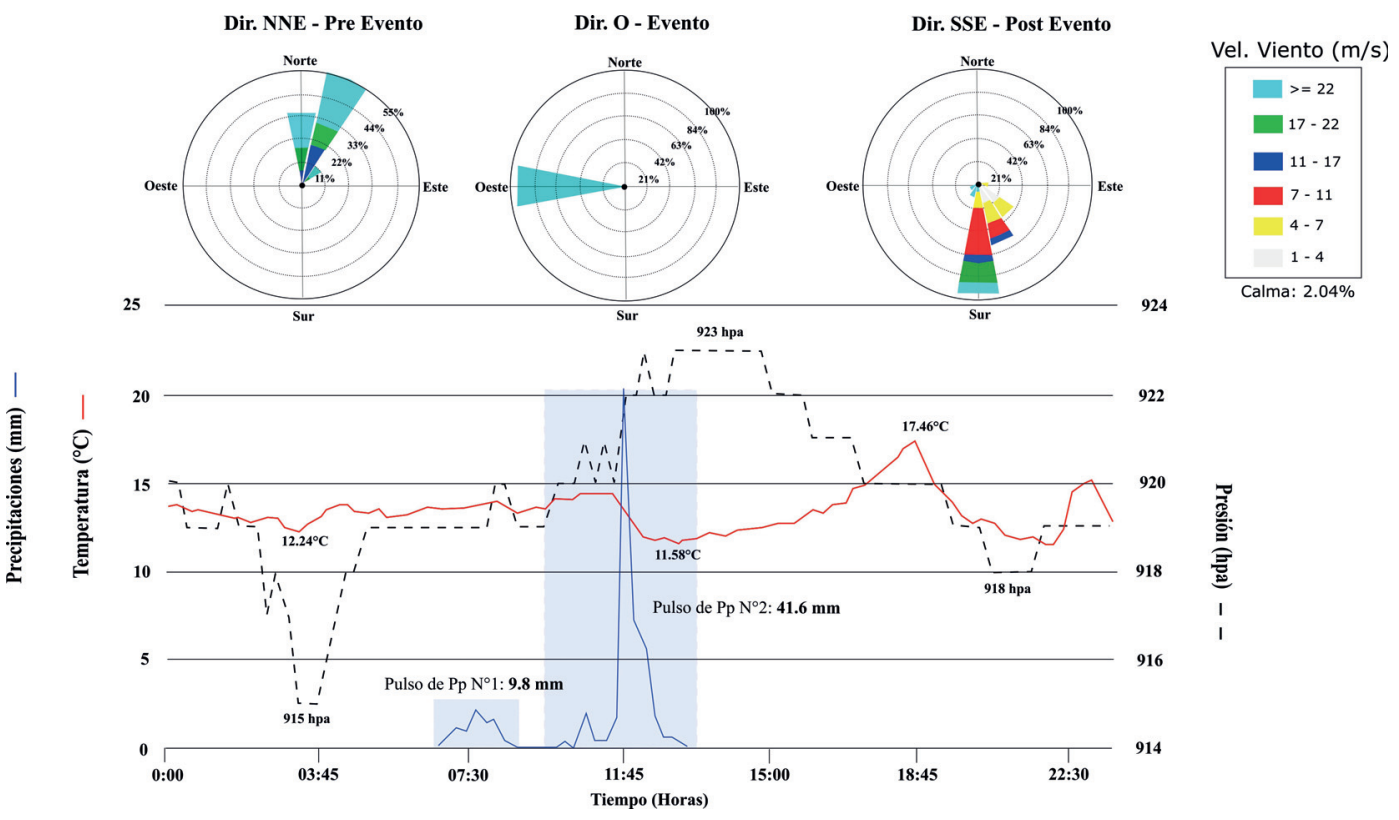

Fuente: Elaboración propia.

\section{Hidrogeomorfología}

En este apartado describimos aspectos de la respuesta hidrogeomorfológica frente a las lluvias descritas en el apartado anterior. El énfasis se pone en la descarga de caudal y su efecto morfogenético en el paisaje. Por un lado, se estimó la erosión de las quebradas № 1 y No 2 al interior de la cuenca de control (Figura $N^{\circ} 3$ ), a partir de la cual se estimó el caudal máximo y el volumen de carga sedimentaria erosionado. Luego se estimó el volumen del relleno sedimentario del cono aluvial depositado a la salida de la cuenca (Figura $N^{\circ} 5$ ).

Las mediciones de las secciones de las quebradas $N^{\circ} 1$ y No 2 permitieron dimensionar la excavación producto del flujo. Esta erosión fue cada vez mayor en dirección hacia la boca de la cuenca, con su parte culminante entre los puntos de control W - Y (Figura No 3), aguas abajo de la confluencia de las quebradas $\mathrm{N}^{\circ} 1$ y $\mathrm{N}^{\circ} 2$, y donde la pendiente alcanza un $10 \%$. El volumen total 
de las secciones transversales excavadas durante el 9A obtenidos a partir de la sumatoria de las mediciones en cada estación de monitoreo (A-Y), alcanzó un valor $1070 \mathrm{~m}^{3}$, que es el valor que se asocia al volumen máximo erosionado en las quebradas monitoreadas (Figura $N^{\circ} 3$ ) como respuesta a la lluvia del 9A. Una parte de este material se depositó en el cono aluvial, dimensionado en un total de $9.455 \mathrm{~m}^{2}$ de superficie. Aquí obtuvimos profundidades de entre 9 y $26 \mathrm{~cm}$ de relleno sedimentario asociado al 9A. Al aplicar la interpolación del tipo kriging de los datos de profundidad obtuvimos una estimación de $637 \mathrm{~m}^{3}$ para el volumen de sedimento acumulado durante el 9A (Figura No 5). Este valor representa el volumen mínimo de sedimentos depositados en el cono aluvial. La descarga hídrica promedio estimada para el curso inferior (entre la confluencia de las quebradas $N^{\circ} 1$ y $N^{\circ} 2$, y el ápice del con aluvial, entre puntos " $\mathrm{P}^{\text {" }} \mathrm{Y}$ " $\mathrm{Y}^{\text {") }}$ alcanzó en promedio unos $11 \mathrm{~m}^{3} \mathrm{~s}^{-1}$ (promedio de los caudales) (Cuadro $\mathrm{N}^{\circ} 1$ ).

Figura No 5

Profundidad y estimación del volumen del cono aluvial a través del método Kriging (ver texto para más detalles). Los puntos con letras ( $B^{\prime}, D^{\prime}$ y $E^{\prime}$ ) corresponden a aquellos utilizados para el estudio de la estratigrafía, además de la profundidad del depósito aluvial 9A. Aquellos puntos sin letra solo fueron utilizados para medir profundidad del depósito. Los puntos $A^{\prime}$ y $C^{\prime}$ no se muestran.

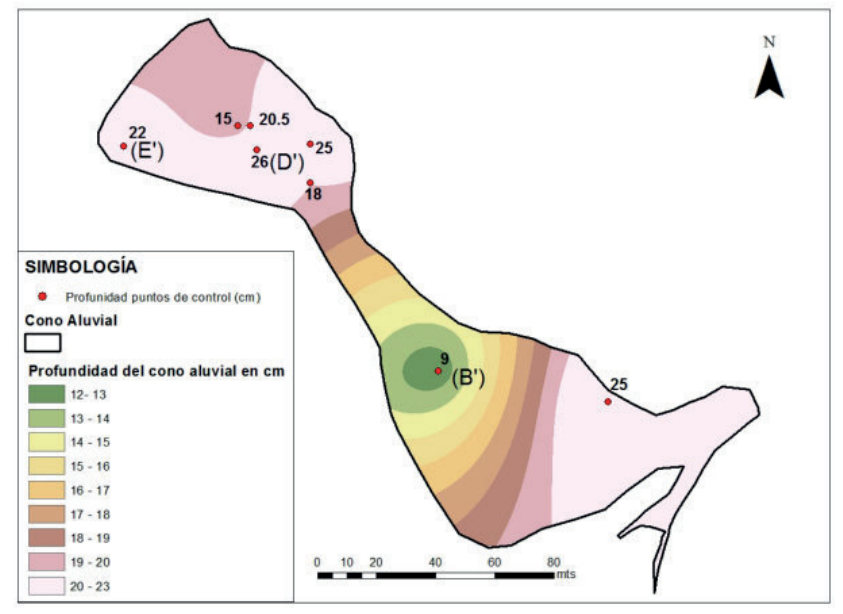

Fuente: Elaboración propia.

Cuadro $\mathrm{N}^{\circ} 1$

Cálculos de Caudal

\begin{tabular}{|l|r|r|r|r|r|r|r|r|r|}
\hline \multicolumn{10}{|c|}{ Quebrada $\mathrm{N}^{\circ} 2$} \\
\hline $\begin{array}{c}\text { Punto de } \\
\text { Control }\end{array}$ & Longitud & Latitud & $\begin{array}{c}\text { Altitud } \\
(\mathrm{m})\end{array}$ & $\begin{array}{c}\text { Área } \\
(\mathrm{m})\end{array}$ & $\begin{array}{c}\text { Perímetro } \\
(\mathrm{m})\end{array}$ & $\begin{array}{c}\text { Radio } \\
\text { Hidráulico }\end{array}$ & $\begin{array}{c}\text { Pendiente } \\
(\%)\end{array}$ & $\begin{array}{c}\text { velocidad } \\
\left(\mathrm{m} \mathrm{s}^{-1}\right)\end{array}$ & $\begin{array}{c}\text { Caudal } \\
\left(\mathrm{m}^{3} \mathrm{~s}^{-1}\right)\end{array}$ \\
\hline A & 380227 & 7696897 & 830 & 0,46 & 1,94 & 0,24 & - & - & - \\
\hline B & 380190 & 7696880 & 826 & 0,54 & 2,1 & 0,26 & 0,1 & 3,2 & 1,7 \\
\hline C & 380163 & 7696860 & 821 & 0,15 & 1,5 & 0,10 & 0,1 & 2,1 & 0,3 \\
\hline
\end{tabular}


Continuación Cuadro No 1

\begin{tabular}{|c|c|c|c|c|c|c|c|c|c|}
\hline \multicolumn{10}{|c|}{ Quebrada No 2} \\
\hline $\begin{array}{c}\text { Punto de } \\
\text { Control }\end{array}$ & Longitud & Latitud & $\begin{array}{l}\text { Altitud } \\
(\mathrm{m})\end{array}$ & $\begin{array}{l}\text { Área } \\
(\mathrm{m})\end{array}$ & $\begin{array}{c}\text { Perímetro } \\
(\mathrm{m})\end{array}$ & \begin{tabular}{|c|} 
Radio \\
Hidráulico
\end{tabular} & $\begin{array}{c}\text { Pendiente } \\
(\%)\end{array}$ & $\begin{array}{c}\text { velocidad } \\
\left(\mathrm{m} \mathrm{s}^{-1}\right)\end{array}$ & $\begin{array}{l}\text { Caudal } \\
\left(\mathrm{m}^{3} \mathrm{~s}^{-1}\right)\end{array}$ \\
\hline D & 380137 & 7696846 & 818 & 0,77 & 2,5 & 0,31 & 0,1 & 3,7 & 2,8 \\
\hline E & 380093 & 7696816 & 815 & 0,45 & 1,9 & 0,24 & 0,1 & 2,3 & 1,0 \\
\hline $\mathrm{F}$ & 380054 & 7696810 & 811 & 0,39 & 1,9 & 0,21 & 0,1 & 2,8 & 1,1 \\
\hline G & 380022 & 7696824 & 807 & 1,16 & 3,5 & 0,33 & 0,1 & 4,0 & 4,7 \\
\hline $\mathrm{H}$ & 379993 & 7696826 & 807 & 1,12 & 3,1 & 0,36 & 0,0 & 0,0 & 0,0 \\
\hline I & 379962 & 7696835 & 800 & 2,42 & 4,4 & 0,55 & 0,2 & 7,8 & 18,9 \\
\hline J & 379933 & 7696834 & 800 & 1,21 & 3,1 & 0,39 & 0,0 & 0,0 & 0,0 \\
\hline \multicolumn{10}{|c|}{ Quebrada No 1} \\
\hline $\begin{array}{c}\text { Punto de } \\
\text { Control }\end{array}$ & Longitud & Latitud & Altitud & $\begin{array}{l}\text { Área } \\
(\mathrm{m})\end{array}$ & $\begin{array}{c}\text { Perímetro } \\
(\mathrm{m})\end{array}$ & $\begin{array}{c}\text { Radio } \\
\text { Hidráulico }\end{array}$ & $\begin{array}{c}\text { Pendiente } \\
(\%)\end{array}$ & $\begin{array}{c}\text { velocidad } \\
\left(\mathrm{m} \mathrm{s}^{-1}\right)\end{array}$ & $\begin{array}{c}\text { Caudal } \\
\left(\mathrm{m}^{3} \mathrm{~s}^{-1}\right)\end{array}$ \\
\hline K & 379958 & 7696759 & 804 & 6,93 & 7,45 & 0,93 & - & - & \\
\hline L & 379946 & 7696767 & 800 & 2,40 & 4,70 & 0,51 & 0,28 & 8,4 & 20,19 \\
\hline M & 379935 & 7696777 & 800 & 2,34 & 4,49 & 0,52 & 0,00 & 0,0 & 0,00 \\
\hline $\mathrm{N}$ & 379923 & 7696786 & 800 & 3,10 & 5,07 & 0,61 & 0,00 & 0,0 & 0,00 \\
\hline$\tilde{\mathrm{N}}$ & 379910 & 7696794 & 798 & 1,89 & 4,10 & 0,46 & 0,13 & 5,4 & 10,21 \\
\hline $\mathrm{O}$ & 379899 & 7696803 & 796 & 3,05 & 5,02 & 0,61 & 0,14 & 6,7 & 20,51 \\
\hline $\mathrm{P}$ & 379889 & 7696813 & 794 & 3,38 & 5,22 & 0,65 & 0,14 & 7,0 & 23,83 \\
\hline Q & 379878 & 7696823 & 792 & 2,19 & 4,19 & 0,52 & 0,13 & 5,9 & 12,98 \\
\hline $\mathrm{R}$ & 379866 & 7696828 & 793 & 2,50 & 4,49 & 0,56 & 0,00 & 0,0 & 0,00 \\
\hline s & 379852 & 7696834 & 790 & 2,39 & 4,41 & 0,54 & 0,20 & 7,4 & 17,67 \\
\hline $\mathrm{T}$ & 379841 & 7696842 & 790 & 1,52 & 3,50 & 0,43 & 0,00 & 0,0 & 0,00 \\
\hline U & 379827 & 7696848 & 790 & 1,46 & 3,55 & 0,41 & 0,00 & 0,0 & 0,00 \\
\hline V & 379814 & 7696854 & 789 & 4,24 & 6,90 & 0,61 & 0,07 & 4,8 & 20,25 \\
\hline W & 379775 & 7696867 & 781 & 1,98 & 5,94 & 0,33 & 0,19 & 5,3 & 10,50 \\
\hline$x$ & 379743 & 7696864 & 780 & 2,05 & 6,71 & 0,31 & 0,03 & 2,0 & 4,11 \\
\hline Y & 379718 & 7696852 & 776 & 4,44 & 10,36 & 0,43 & 0,14 & 5,4 & 23,99 \\
\hline \multicolumn{10}{|r|}{11.33} \\
\hline
\end{tabular}

Fuente: Elaboración propia.

\section{Estratigrafía}

La estratigrafía incluyó la descripción de sedimentos en el cono aluvial 9A y en las quebradas $\mathrm{N}^{\circ} 1$ y $\mathrm{N}^{\circ} 2$ excavadas durante la descarga hídrica y exponiendo sedimentos antiguos. En ambos casos se confeccionaron columnas estratigráficas (Figuras $N^{\circ} 6, N^{\circ} 7, N^{\circ} 8, N^{\circ} 9$ y $N^{\circ} 10$ ) que posteriormente fueron correlacionadas para obtener una secuencia tipo para ambos casos (discusión). 


\section{Cono Aluvial}

Para facilitar la descripción del depósito aluvial se dividió el cono en tres áreas de acuerdo a su posición: proximal (zona I), media (zona II) y distal (zona III). Se realizaron un total de cinco calicatas (columnas estratigráficas) para describir y correlacionar la estratigrafía aluvial del depósito (Figura No 3 y No 7 ).

- Columna estratigráfica A' (20049' 27,93" S, $70^{\circ} 09^{\prime} 21,40^{\prime \prime}$ O, 784 m s.n.m.)

Corresponde a un perfil expuesto de $55 \mathrm{~cm}$, localizado en la parte proximal del depósito aluvial, cerca del ápice (Figura № 6).

Se distinguen tres unidades de litofacies, que son descritas a continuación de base a techo:

U1: Corresponde a unidad de arena media de $2 \mathrm{~cm}$ de espesor. Esta arena tiene una mediana a mala selección. Las raíces finas son comunes. Las raíces preservadas denotan la antigua germinación de plantas asociadas a una pedogénesis muy incipiente.

U2: Corresponde a una brecha sedimentaria con una potencia de $39 \mathrm{~cm}$, y estratificación gruesa. La grava aparece clasto soportada, monomíctica, con clastos subangulosos con una esfericidad subprismática. La mayoría de los clastos son de 7 a $10 \mathrm{~cm}$ en su eje mayor. Estos se presentan con diversas orientaciones, pero con una preferencia clara de flujo de este a oeste (correspondiente a la dirección de la pendiente). Por otra parte, tiene presencia de clastos con una pátina o lamina de carbonato en una de sus caras.

U3: Corresponde a un estrato de arena fina masiva, de $2 \mathrm{~cm}$ de espesor.

Figura No 6

Columna estratigráfica del punto de control $A^{\prime}$ en el depósito aluvial (ver localización y leyenda en la Figura $N^{\circ} 7$ )

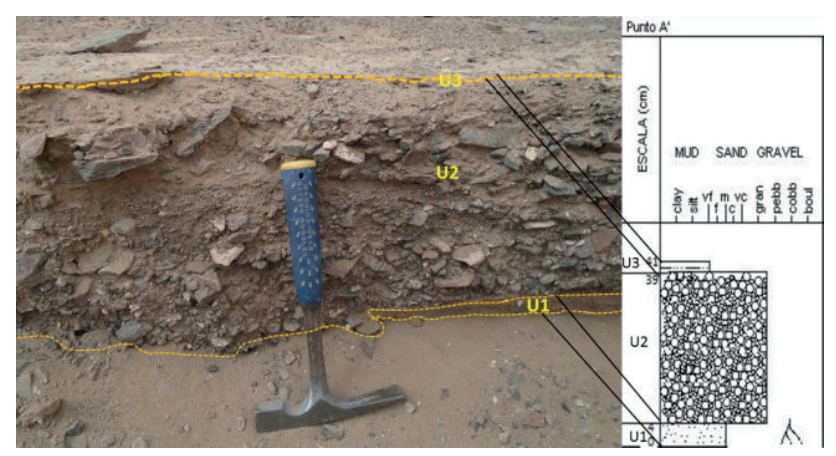

Fuente: Elaboración propia.

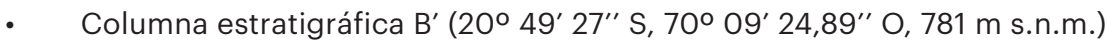


Corresponde a una calicata de $37 \mathrm{~cm}$ de profundidad, que se encuentra localizada en la posición media del cono aluvial. Se distinguen tres unidades de litofacies, de abajo hacia arriba:

U1: Posee una potencia de $24 \mathrm{~cm}$, correspondiente a una arena media, de moderada selección. Presencia de una capa de raíces gruesas en el contacto abrupto con la unidad superior. Raíces finas abundan en el interior del sedimento igualmente, además de conchas de gastrópodo terrestre de la especie Bostrix delerictus Broderip. La arena ha perdido parte de su estructura primaria pero aún conserva rastros de una laminación sedimentaria original.

U2: Posee una potencia de $9 \mathrm{~cm}$. Es un estrato arenolimoso, que consiste en varias secuencias laminadas de sedimentos finos y gruesos. Presencia de Bostrix delerictus Broderip.

U3: Capa masiva de arena fina masiva, con potencia de $4 \mathrm{~cm}$.

- Columna estratigráfica $C^{\prime}\left(20^{\circ} 49^{\prime} 26,50^{\prime \prime} \mathrm{S}, 70^{\circ} 09^{\prime} 25,58^{\prime \prime} \mathrm{O}, 780\right.$ m s.n.m. $)$

Corresponde a una calicata, que se encuentra localizada en la parte media del cono aluvial, aguas debajo de $\mathrm{B}^{\prime}$. Posee una potencia total de $37 \mathrm{~cm}$.

U1: Tiene una potencia de $10 \mathrm{~cm}$. Es un estrato de arena muy gruesa, medianamente seleccionada. Gravas monomícticas, semiangulosas a subredondeadas ocurren en esta unidad. El conjunto sedimentario presenta una tendencia granocreciente. Presencia de raíces finas.

U2: Posee una potencia de $21 \mathrm{~cm}$. Brecha sedimentaria clasto soportado y gruesamente estratificada. Los clastos son de tamaño máximo $5 \mathrm{~cm}$ y media de $3 \mathrm{~cm}$, subangulosos, y están orientados preferentemente en dirección este a oeste (dirección de la pendiente). Estos clastos son monomícticos. Presencia de concha rota de gastrópodos terrestres, del mismo tipo que en las otras columnas sedimentarias descritas. El contacto con la unidad superior es abrupto.

U3: Posee un espesor de $6 \mathrm{~cm}$. Esta unidad corresponde a arena fina masiva.

- Columna estratigráfica D' $\left(20^{\circ} 49^{\prime} 25,17^{\prime \prime} \mathrm{S}, 70^{\circ} 09^{\prime} 26,88^{\prime \prime} \mathrm{O}, 777\right.$ m s.n.m.)

Corresponde a una calicata en la parte distal del cono aluvial y posee una potencia de $49 \mathrm{~cm}$.

U1: Tiene un espesor de $20 \mathrm{~cm}$. Es una arena media, bien seleccionada, con presencia de escasos guijarros de andesita de la formación Oficina Viz, que es la misma que aparece en las otras unidades. Estos clastos son subangulosos a subredondeados y con una esfericidad subprismática. Este estrato tiene presencia de raíces y del gastrópodo terrestre Bostrix delerictus Broderip. El contacto con la unidad superior es nítido.

U2: Posee una potencia de $26 \mathrm{~cm}$. Este estrato corresponde a una arena media, bien seleccionada y bien laminada. Se incluyen algunos clastos aislados flotando en la matriz, los cuales son subangulosos y de c. $7 \mathrm{~cm}$ en su eje mayor. Su orientación pareciera indicar la dirección preferencial del flujo. Presencia de Bostrix delerictus Broderip. Se diferencia del estrato subyacente por un menor grado de compactación. Además, posee tres láminas de limo y arcilla, que aparecen intercaladas en el estrato arenoso. 
U3: Estrato de arena fina masiva que tiene un espesor de $3 \mathrm{~cm}$ y se depositó con contacto abrupto sobre la unidad inferior.

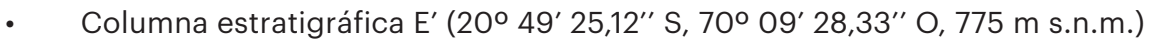

Este corresponde a una calicata en la zona distal del aluvión. Posee una potencia de $46 \mathrm{~cm}$.

U1: Tiene una potencia de $31 \mathrm{~cm}$. Corresponde a una arena media, medianamente seleccionada, con presencia de clastos de dimensiones de guijarro, los cuales son subangulosos y de esfericidad prismático y esférico. Estos clastos son monomícticos y se encuentran inserto en una matriz. El aspecto del sediento es masivo. Este estrato posee raíces medias a finas.

U2: Posee un espesor de $12 \mathrm{~cm}$. Es una arena media a fina, que posee una tendencia granodecreciente. Este estrato en su techo está formado una capa limo arcillosa, que acentúa la laminación del sedimento.

U3: Estrato de arena fina masiva de $3 \mathrm{~cm}$ de potencia. El contacto con la unidad inferior es nítido.

En relación al conjunto estratigráfico descrito para el cono aluvial 9A (Figura № 7), se aprecian de manera generalizada tres unidades sedimentarias que van desde las gravas a limos arcillosos. Unidades de gravas carentes de raíces sobreyacen y subyacen arenas y finos. Paralelamente, en la parte proximal del cono aluvial hay dos subunidades no descritas en las secciones presentadas: unidades $\mathrm{U} 2 \mathrm{a}$ y $\mathrm{U} 2 \mathrm{~b}$, que muestran una tendencia textural diferente, con los gruesos sobreyaciendo a los finos (Figura No $8 \mathrm{C}$ ), aspecto que discutimos más abajo. Importante, la presencia de raíces sólo ocurre en unidades estratigráficamente inferiores y están ausente en las superiores, lo que da un marco estratigráfico de referencia para identificar el evento $9 \mathrm{~A}$.

Figura No 7

Correlación de las secuencias litoestratigráficas del cono aluvial 9A en Alto Patache, distinguiendo sus zonas proximal, media y distal. Se propone una relación estratigráfica entre las columnas descritas.

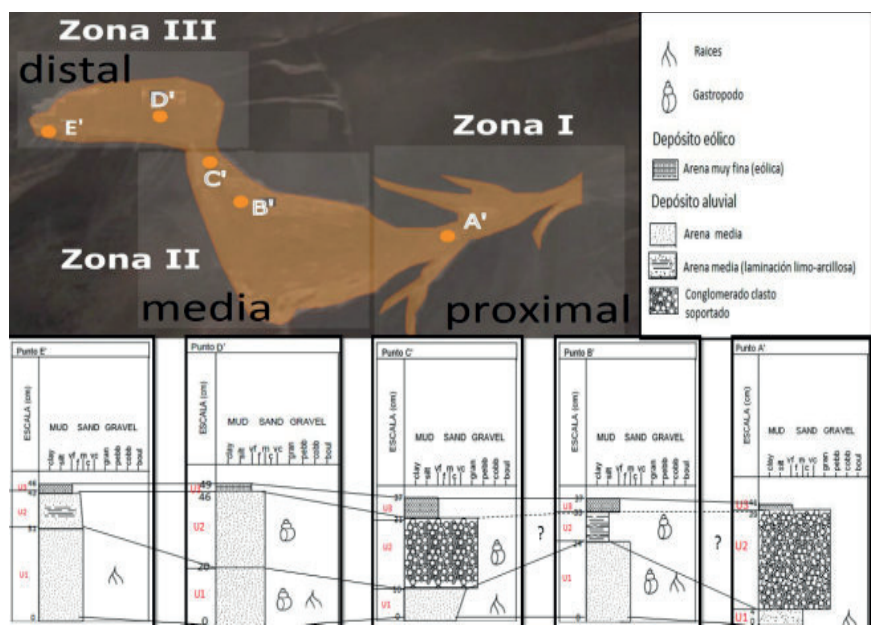

Fuente: Elaboración propia. 
Figura No 8

Cono aluvial del 9 de agosto. A Gravas y guijarros de la columna C, que están asociados a la unidad 2. B Grietas de secamiento en limo y arcilla, en la parte distal del cono aluvial. C Estratigrafía en un perfil expuesto en la parte proximal del cono aluvial no descrito como columnas en el texto; en ella se reflejan dos subunidades: U2a y U2b, la primera arena media con intercalaciones de láminas limoarcillosas, la segunda corresponde a gravas y guijarros. D Sedimentos finos de la parte distal del cono aluvial, el cual está asociado a un estrato de arenas medias a finas con intercalaciones de láminas limo-arcillosas de la columna $\mathrm{E}^{\prime}$

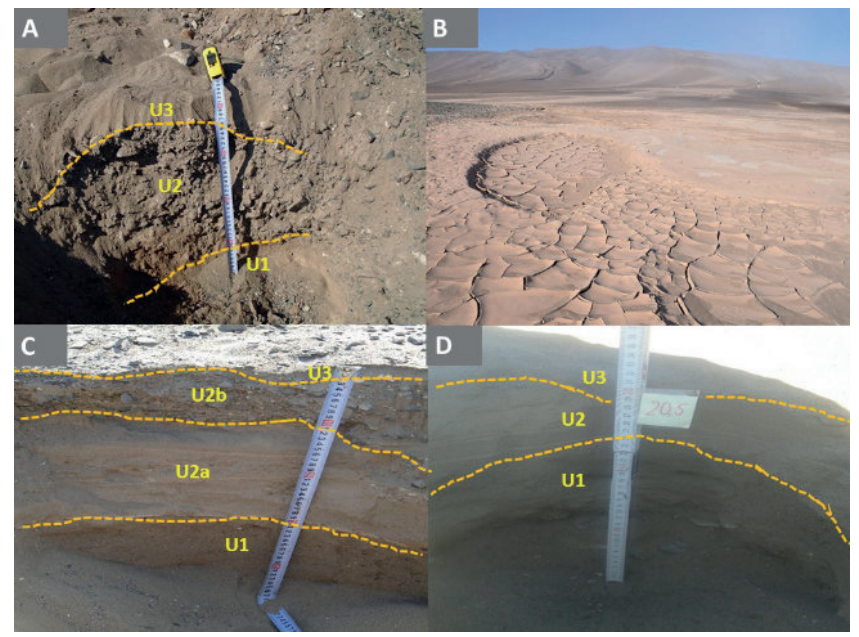

Fuente: Elaboración propia.

\section{Quebradas}

En relación a la estratigrafía de las quebradas describimos a continuación las columnas A, I, K, P (Figura No 3).

\section{- Columna estratigráfica A (200 49' 26" S, $70^{\circ} 09^{\prime} 03^{\prime \prime}$ O, 834 m s.n.m.)}

Corresponde al primer punto de la cabecera de la cuenca, en la quebrada 2. Este perfil expuesto posee una potencia de $55 \mathrm{~cm}$ (Figura No 9). La sección sedimentaria puede ser dividida en dos litofacies que de base a techo de la secuencia son:

U1: Su espesor máximo es de $28 \mathrm{~cm}$. Corresponde a un tipo de carbonato masivo, dada su fuerte reacción al $\mathrm{HCl}$. Es un estrato bien compacto y de color blanquecino. Su techo está en discordancia erosiva con el estrato suprayacente.

U2: Su potencia es de $27 \mathrm{~cm}$. Esta facie corresponde a un estrato de arena aluvial fina con un grado de compactación intermedio, que tiene presencia de clastos esporádicos matriz soportados. El sedimento es aparentemente masivo y los clastos caídos dejan sus oquedades en el sedimento. Los clastos poseen tamaños polimodales, en promedio $8 \mathrm{~cm}$ en su eje mayor, y un máximo de $11 \mathrm{~cm}$. Su forma es mayormente sub-angulosa a sub-redondeada y una esfericidad entre sub-prismático y esférico. Los clastos son monomícticos. El sedimento tiene raíces actuales y conchas de Bostrix delerictus Broderi. 
Figura No 9

Columna estratigráfica $A$ en la quebrada No2. (ver leyenda en la Figura No 10)

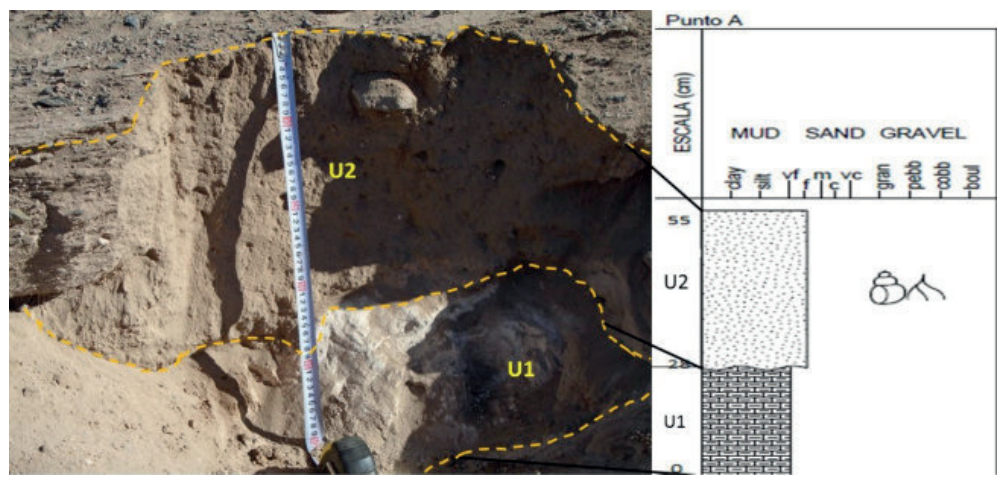

Fuente: Elaboración propia.

- Columna estratigráfica I (200 49' 28" S, $70^{\circ} 09^{\prime} 12^{\prime \prime} \mathrm{O}, 812$ m s.n.m.).

Corresponde a un perfil expuesto de la quebrada No $2 \sim 80 \mathrm{~m}$ río arriba de la confluencia con la quebrada No 1. Posee una potencia de $27 \mathrm{~cm}$.

Sus unidades de litofacies de base a techo son:

U1: Corresponde a un estrato de gravas polimodales y monomícticas de Andesitas de Oficina Viz. Posee una mala selección. Sus clastos son subangulosos, sub-prismáticos y se encuentran en su mayoría clasto-soportados. El tamaño de los clastos puede llegar a $6 \mathrm{~cm}$.

U2: Es un depósito de limólita, con alta presencia de carbonato de color blanquecino que reacciona fuertemente al contacto con el $\mathrm{HCl}$. Posee clastos de tamaños centimétricos tipo grava a pedrusco, que no tienen una orientación preferencial. Hay presencia de raíces actuales y estructuras de bioturbaciones de raíces. Su parte superior grada nítidamente al pavimento desértico que caracteriza la superficie actual del entorno.

- Columna estratigráfica K (200 49' 30" S, $70^{\circ} 09^{\prime} 13^{\prime \prime} \mathrm{O}, 807$ m s.n.m.)

Corresponde a la cabecera actual de la quebrada No 1. Posee una potencia de $120 \mathrm{~cm}$.

Sus unidades de litofacies de base a techo son:

U1: Tiene una potencia de $55 \mathrm{~cm}$. Corresponde a un estrato de arena muy gruesa, gravosa, la cual posee clastos de dimensiones de tipo guijarro. Los clastos poseen una redondez sub-angulosa a sub-redondeada, además de una esfericidad entre sub-prismático y esférico. Estos se encuentran formando estratos más o menos definidos, aspecto que denota la abundancia de matriz que soporta los clastos. Su disposición cercana a la horizontal. La arena no presenta estructura obvia y es más bien masiva. Hacia la base de la unidad se desarrolla una lámina blanquecina, que reacciona al contacto con $\mathrm{HCl}$, de lo que se deduce su contenido de carbonato. El contacto con la unidad superior es nítido. 
U2: Su espesor máximo es de $65 \mathrm{~cm}$. Esta facie corresponde se compone de dos sub-unidades en contacto nítido. Ambas de arena media diferenciados por la tonalidad de las mismas, siendo grisácea la inferior y pardo la superior. La arena posee clastos esporádicos de 7 a $13 \mathrm{~cm}$, subangulosos y subredondeados y de esfericidad del tipo sub-prismático a esférico. Los clastos son monomícticos y polimodales. Tiene presencia de raíces abundantes, bulbos de plantas actuales e individuos muertos de Bostrix delerictus Broderip. En cuanto a la sub-unidad suprayacente desde esta misma facie es una arena media de color pardo con clastos flotando en la matriz. Los clastos son de redondez sub-angulosa a sub-redondeada y de esfericidad entre sub-prismático y esférico. Hay presencia de raíces y de individuos de Bostrix delerictus Broderip.

- Columna estratigráfica $\mathrm{P}\left(20^{\circ} 49^{\prime} 29^{\prime \prime} \mathrm{S}, 70^{\circ} 09^{\prime} 15^{\prime \prime} \mathrm{O}, 803 \mathrm{~m}\right.$ s.n.m. $)$

Corresponde a una columna de la quebrada $\mathrm{N}^{\circ}$ 1, en la confluencia con la quebrada $\mathrm{N}^{\circ} 2$ (Figura $\mathrm{N}^{\circ}$ 3). Posee una potencia de $105 \mathrm{~cm}$.

Sus unidades de litofacies de base a techo son:

U1: Posee una potencia de $50 \mathrm{~cm}$. Esta facie corresponde a una brecha sedimentaria, cementada con yeso, conformada por clastos monomícticos de la Formación Oficina Viz (Andesita), que son polimodales y de tamaño promedio del tipo guijarro. Sus clastos son subangulosos y de esfericidad subprismática y esférica. El sedimento es clasto soportado y muestra estratificación.

U2: Posee una potencia de $55 \mathrm{~cm}$. Esta facie corresponde a un estrato de areno gravoso, con clastos flotando en la matriz arenosa. La concentración de clastos varía lateralmente y aparecen bolsones arenosos que pueden contrastar con agrupaciones de gravas. La estratificación de esta unidad no es obvia. Los clastos alcanzan dimensiones tipo guijarro, con redondez sub-angulosa a subredondeada y de esfericidad del tipo sub prismático y esférico. Tiene presencia de raíces recientes, abundantes, junto a conchas de gastrópodos terrestre de Bostrix delerictus Broderip.

Figura No 10

Correlación de las secuencias litoestratigráficas de las quebradas profundizadas durante el 9A en Alto Patache.

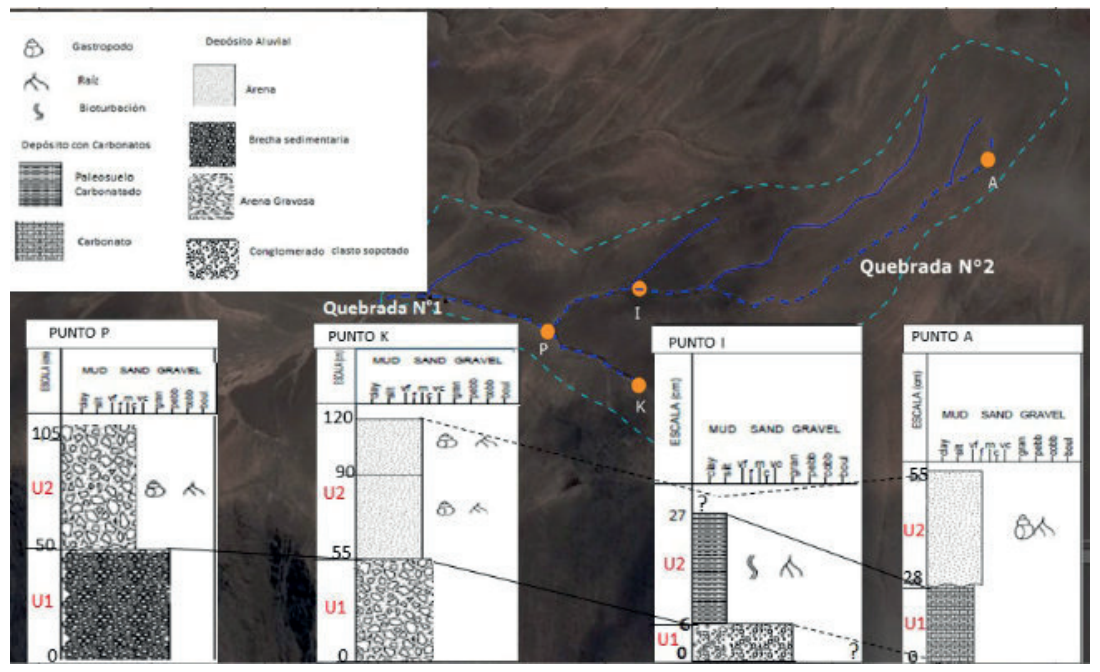

Fuente: Elaboración propia. 
Figura No 11

Facies sedimentarias presentes en las quebradas estudiadas, incluyendo secciones descritas como columnas estratigráficas en el texto. Las unidades $N^{\circ} 1$ y $N^{\circ} 2$ hacen referencia a la estratigrafía tipo interpretada para el sector (Figura No 10). A, muestra la cabecera de la quebrada No 1 y la dinámica de erosión retrograda del evento $9 \mathrm{~A}$. De aquí proviene la columna $\mathrm{K}$. B, perfil expuesto próximo a la columna $\mathrm{K}$ de la quebrada $\mathrm{N}^{\circ} 1$, en donde se ve la unidad 2, asociada a un ambiente aluvial. C, gravas y guijarros, asociados a facies aluviales de la unidad 1. Nótese la lente de la unidad 1 que aparece rodeada por arenas de la unidad 2. Foto tomada entre los puntos de control S y R, quebrada No 1

(Figura No 3). D, Gravas y guijarros de la unidad 1 aluvial en la columna Q (quebrada N²).

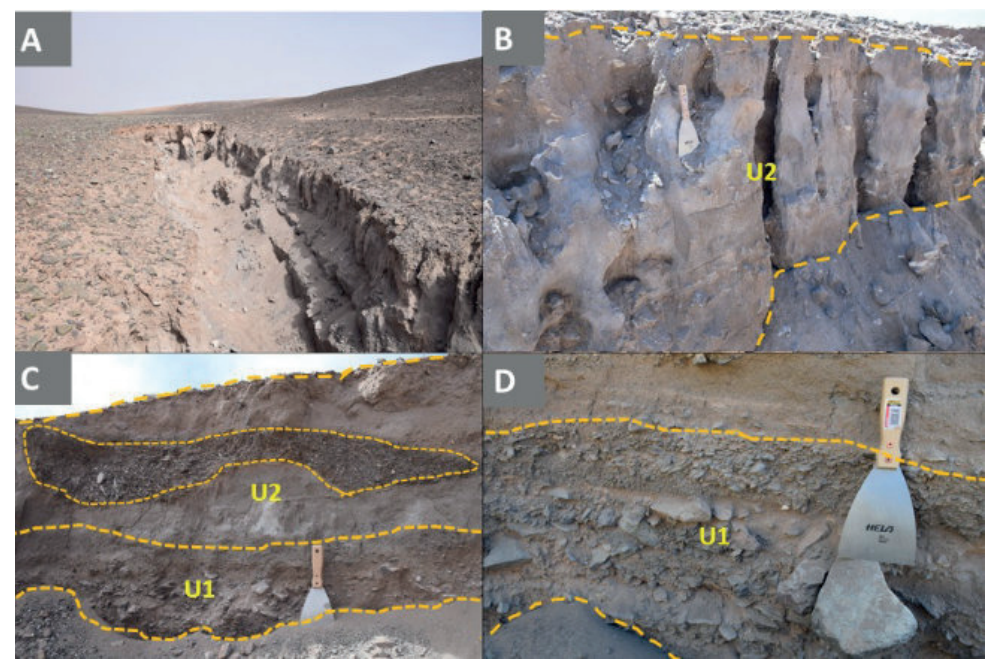

Fuente: Elaboración propia.

En síntesis, el conjunto estratigráfico expuesto en las quebradas $N^{\circ} 1$ y № 2 muestra unidades gravosas estratificadas y otras arenosas con clastos soportados, pasando por unidades masivas carbonatadas, todas ellas representan variaciones ambientales que discutimos más abajo.

\section{Dinámica y evolución de los eventos hidrometeorológicos y geomorfológicos del 9 de agosto de 2015 en Alto Patache}

\section{Extensión y causa de las lluvias del 9A}

Durante la madrugada del 9 de agosto 2015 hubo una lluvia de carácter torrencial en Alto Patache y otros sectores de la cordillera costera de la región de Tarapacá. Entre las 07:00 y las 13:00 horas, cayeron un total de 51,4 mm en Alto Patache. La Figura № 4 muestra que el desarrollo del evento de precipitación estuvo marcado por dos peaks, uno menor entre las 07:00 y las 10:00 horas y otro principal entre las $10: 30$ y las 13:00 horas, cuando cayeron $9,8 \mathrm{~mm}$ y 41,6 $\mathrm{mm}$, respectivamente. La lluvia fue anticipada por el paso abrupto de una depresión atmosférica, que trajo cambios en la dirección e intensidad del viento en las siguientes horas. El peak de la 
precipitación ocurrió con viento Oeste y velocidades de 17,3 $\mathrm{m} \mathrm{s}^{-1}$. Al mismo tiempo que Alto Patache recibía el embate de las lluvias, Tocopilla $100 \mathrm{~km}$ al sur, era afectada por el mismo evento. Aquí entre las 5-8 horas se midieron 12,6 mm, condición que generó, como en Alto Patache, la activación de quebradas y aluviones (Grijalba, 2016). Esta precipitación se debió a una vaguada profunda que abarcó varios sectores del norte de Chile durante el 9 de agosto, hasta cerca de Iquique por el norte (Figura No 12). Grijalba (2016) describe que la vaguada profunda se formó por la unión de dos bajas al norte y sur de Chile, entre dos altas, una al oeste sobre el Pacífico y la otra en el Atlántico (Figura No 12). La humedad de esta vaguada en este caso provino del oeste en latitudes medias y, en vez de desviarse hacia el sur (como debía suceder por el Anticiclón del Pacifico Sur), siguió trayectoria definida por las altas y bajas de la vaguada profunda, llegando al Norte Grande de Chile y generando la precipitación observada (Figura No 12A) (Grijalba, 2016). Por otro lado, es importante destacar, que la anomalía de precipitación primero llegó a las latitudes de Chile central el 7 de agosto, desplazándose luego hacia el norte de Chile $\left(20^{\circ}-25^{\circ} \mathrm{S}\right)$, entre el 8 y 9 de agosto (Figura $N^{\circ} 12 B$ ). En este último caso (9A), llegando al peak de la tormenta, el cual se registró instrumentalmente entre Tocopilla y Alto Patache Patache $\left(22^{\circ}-20^{\circ} \mathrm{S}\right)$. En Alto Patache,

Figura No 12

La tormenta del 9A en el Norte Grande. A, Re-análisis para la anomalía de altura geopotencial para los días 8-9 de agosto de 2015. Izquierda $500 \mathrm{mb}$, derecha $1000 \mathrm{mb}$. A: alta presión de la vaguada profunda. B: baja presión de la vaguada profunda. B, Re-análisis de anomalía de Tasa de Precipitación ( $\mathrm{mm} / \mathrm{día}$ ) para los días 7-8-9 de agosto de 2015. Los colores fríos indican anomalía positiva. Flecha indica el transporte de la precipitación, y el contorno encierra las precipitaciones de la tormenta.

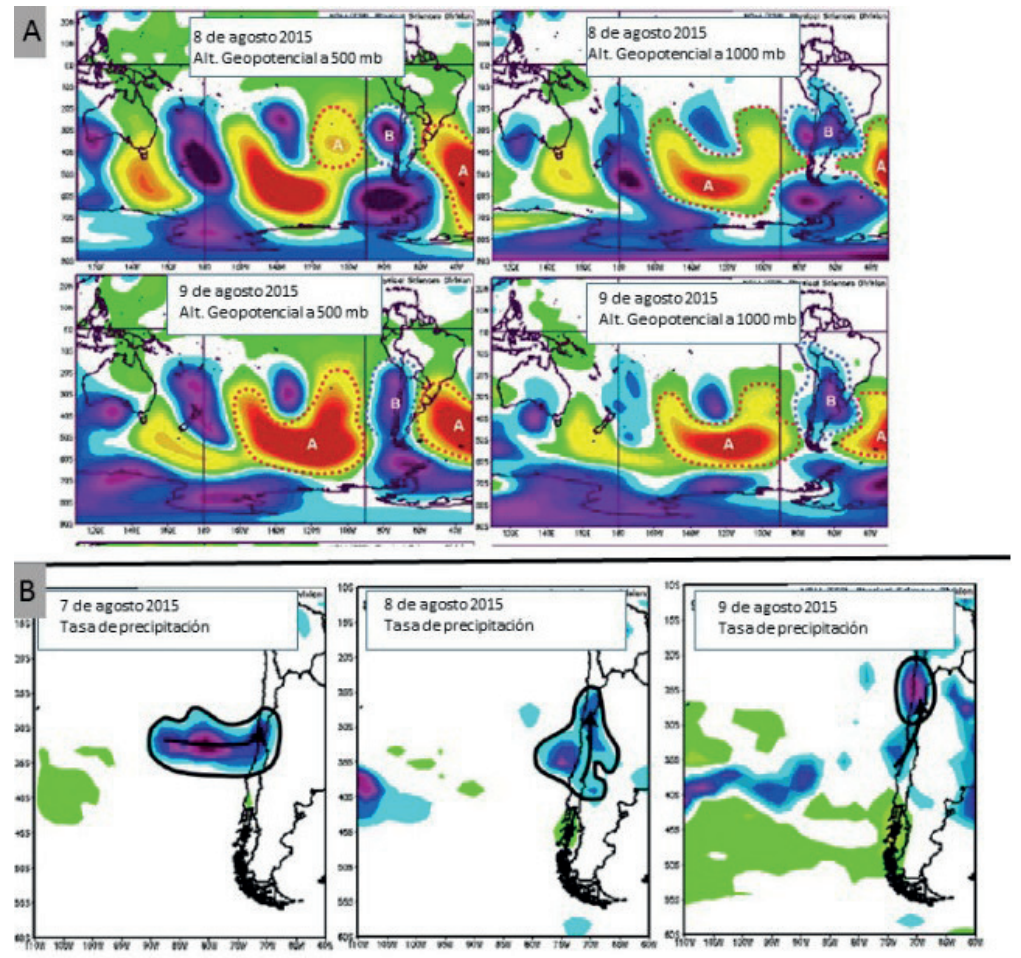

Fuente: Modificado de Grijalba (2016). 
la cantidad e intensidad de la precipitación fue mayor que la registrada en Tocopilla, dado que precipitó entre las 07:00 y las 13:00 horas, cayendo un total de 51,4 mm versus $12,6 \mathrm{~mm}$, divididos en dos pulsos de lluvia. Se deduce que la precipitación tipo tormenta de alta intensidad y corta duración abarcó un área espacial acotada con una dinámica convectiva, es decir, las lluvias se hicieron sentir en zonas acotadas del desierto costero, aspecto que pudo observarse también en la carencia de erosión hídrica en zonas aledañas a Alto Patache.

\section{El aluvión del 9A en Alto Patache}

La estimación de erosión y depositación de sedimentos, además de descarga hídrica asociada al 9A, debe ser tomada como una aproximación. Según el cálculo de erosión asociado a la incisión de quebradas (c. $1070 \mathrm{~m}^{3}$ ) es muy superior al volumen de sedimentos depositados en el cono aluvial desarrollado a partir de la boca de la cuenca (c. $640 \mathrm{~m}^{3}$ ). Por ende, pareciera estar sobrevalorando la real erosión ocurrida en las quebradas de estudio. Una probable razón de esta diferencia es que los datos utilizados no consideran la dimensión original de las quebradas, las que ya tenían algún grado de incisión pre $9 \mathrm{~A}$, particularmente en su curso medio y bajo. Al mismo tiempo, es probable que una parte del sedimento no se depositó en el cono aluvial y fue transportado pendiente abajo hacia el acantilado costero. De todas maneras, el valor mínimo de c.640 m³ calculados para el depósito aluvial es muy significativo para una cuenca menor como la analizada en este estudio; este valor equivale a 64 tolvas de $10 \mathrm{~m}^{3}$ de carga producidos a partir de una cuenca de 7,2 ha. Por su parte, el volumen de descarga hídrica estimado a partir de la aplicación del método de Manning, que considera la dimensión de la incisión (sección mojada), la rugosidad del canal de escorrentía y la pendiente, produjo valores que variaron entre 1,1 y $49 \mathrm{~m}^{3} \mathrm{~s}^{-1}$ (Cuadro $N^{\circ}$ 1). Los valores de caudal menores se obtuvieron en la cabecera de la quebrada $N^{\circ} 2$, donde las secciones transversales tienen menor área $\left(0,3 \mathrm{~m}^{2}\right)$. Los valores mayores se obtuvieron en la cabecera de la quebrada № 1, donde el área de la sección mojada alcanzó 4,7 m². No obstante, el valor más representativo de la descarga total durante el aluvión debe ser próximo al valor medio del tramo de la quebrada río abajo de la confluencia de las quebradas $\mathrm{N}^{\circ} 1$ y $\mathrm{N}^{\circ} 2$. Este valor es de c.11 $\mathrm{m}^{3} \mathrm{~s}^{-1}$, caudal que fluyó con un gran poder erosivo y una competencia tal que logró transportar clastos de hasta $17 \mathrm{~cm}$ en su eje mayor, que fueron depositados en la parte alta del cono aluvial. Muchos de estos clastos fueron erosionados desde la cabecera de la quebrada $N^{\circ} 1$, aspecto que se evidencia por la capa de carbonato de los clastos, los que abundan en las exposiciones de la parte alta de esta quebrada. Se deduce entonces que el flujo fue capaz de transportar todo lo que encontró a su paso. Situación similar ocurrió en Tocopilla, donde los aluviones costeros cortaron la carretera, interrumpieron servicios y dejaron cerca de 4000 damnificados (Grijalba, 2016).

\section{Sedimentología del aluvión 9A en Alto Patache}

La correlación de las facies sedimentarias estudiadas en cada columna estratigráfica permite generar una estratigrafía tipo, tanto para el cono aluvial (aluvión 9A) como para las quebradas estudiadas (pre-aluvión 9A; ver sección siguiente). En el caso del cono aluvial, la unidad basal de arenas medias gravosas, monomícticas, que poseen raíces y presencia de conchas de gastrópodos, se asocia a un periodo de estabilidad con algún grado de pedogénesis mínima sobre sedimentos transportados pre aluvión 9A. Sobre esta unidad le sigue el depósito aluvial del 9A propiamente tal, cuya unidad basal consiste en gravas y pedruscos monomícticos subangulosos, en la cual pueden existir dos subunidades (U2a y U2b) como en el caso del ápice del cono aluvial (Figura $\mathrm{N}^{\circ} 8 \mathrm{C}$ ), en donde queda en evidencia dos pulsos: uno de flujo de barro (U2a) y sobre este 
un flujo detrítico (U2b). Sobreyacente a U2, se expone la U3 que corresponde en algunos casos a un deposito aluvial de baja energía y en otros a un depósito eólico post 9A (Figura № 7).

Las facies sedimentarias estudiadas en cada columna estratigráfica del cono aluvial evidencia las características de transporte y de flujo del aluvión de la mañana del $9 \mathrm{~A}$ en Alto Patache. Las unidades basales incluyen raíces y Bostrix delerictus Broderip, las primeras no presentes en las unidades superiores y por ende identifican la antigua superficie pre-evento 9A. A partir de esta superficie identificamos entonces la capa aluvional 9A. Esta aproximación coincide con las profundidades medidas a partir de los tubos PCV enterrados por el aluvión. Se aprecia que el conjunto de la estratigrafía incluye unidades sedimentarias cuyas texturas se encuentran entre los guijarros y limos arcillosos. El aluvión 9A incluye una capa más gruesa en la base y una más fina suprayacente. Por ejemplo, en las columnas de las zona alta y media del cono aluvial predominan gravas que gradan a arenas (columnas $A^{\prime}$ y $C^{\prime}$, Figura $N^{\circ} 7$ ), mientras que en las columnas de la zona distal del cono predominan las arenas intercaladas con limos arcillosos y que pueden gradar a arcillas en superficie (puntos $E^{\prime}$ y $D^{\prime}$, Figuras $N^{\circ} 7$ ). De esta forma, el análisis sedimentario muestra dos aspectos fundamentales. Primero, que existe la clásica correlación granulométrica de más grueso a más fino entre las partes proximales y distales del cono aluvial, respectivamente, como se describe en todo depósito de este origen (e.g. McGowen \& Groat, 1971) (Figura No 7). Segundo, en cada columna estratigráfica se observa una tendencia granodecreciente de la unidad 2 a la unidad 3 (Figura $N^{\circ} 7$ ), que es el depósito actual, a excepción de la columna B', que puede denotar evidencia de la sedimentación más distal (sedimentación fina) de un cono aluvial más pequeño, formado en la primera fase de precipitación y que tuvo un comportamiento de tipo flujo de barro, el cual posteriormente fue cubierto por un gran flujo de detrito en respuesta al segundo y principal pulso de precipitación en Alto Patache (Figura No 8C).

\section{Anatomía del aluvión 9A en Alto Patache}

El contraste entre la meteorología y la estratigrafía del depósito aluvial 9A nos permite reconstruir integralmente la estructura de este evento registrado en una cuenca de Alto Patache, incluyendo tres fases distintivas (Figura $N^{\circ} 13$ ). Fase Inicio, desarrollada entre las 07:00 y las 10:00 horas, cuando se registró un primer pulso de precipitación de 9,8 $\mathrm{mm}$. En esta fase se produjo el comienzo de la erosión en las laderas e incisión parcial de las quebradas. La presencia de una capa tipo pavimento desértico en la superficie de Alto Patache, con alto contenido de carbonato cementando las arenas superficiales, debió de haber propiciado una rápida respuesta hidrológica luego de haberse superado la capacidad de infiltración del suelo (Haug et al., 2010). Por ende, en esta fase es muy probable que las aguas fluyeron por las laderas primero laminarmente y luego en pequeños surcos que lograron activar las quebradas preexistentes. Fase Peak, ocurrió entre las 10:30 - 13 horas, cuando se registró un total de 41,6 mm de lluvias con un máximo de 20,4 mm en 15 minutos (Figura No 4). Frente a esta intensidad de lluvias, caídas sobre un suelo probablemente saturado desde la Fase Inicio, la escorrentía superficial fue instantánea. El caudal alcanzo su máximo, que estimamos en promedio de $11 \mathrm{~m}^{3} \mathrm{~s}^{-1}$ en el curso inferior de la cuenca. Se generó así la incisión principal de las quebradas, la erosión de materiales y su transporte hasta el depósito aluvial. Las granulometrías mayores (cantos, sub bloques) fueron erosionadas y transportadas en esta fase. Por ejemplo, aquellos clastos con una capa de carbonato blanco presentes en la estratigrafía de la cabecera de la quebrada $N^{\circ} 1$ (puntos $K, L, M, \tilde{N}$ ), fueron mapeados en la parte proximal de la superficie del cono aluvial. Las unidades sobreyacentes (unidad 2), a las basales 
(unidad 1) del cono aluvial (gravas en los puntos $A^{\prime}$ y $C^{\prime}$ y arenas en los puntos $D^{\prime}-E^{\prime}$, unidad 2) fueron depositadas durante esta fase. En esta fase también se generó una potente excavación retrógrada de la quebrada No 1 (Figura No 11A). Fase Término, se desarrolló a partir de la culminación de las precipitaciones, después de las 13:00 horas, y consistió en un gradual decrecimiento de la descarga hídrica en las quebradas, inexistencia de erosión y por sobretodo depositación de la capa fina superior en el cono aluvial, que fue luego cubierta por la capa eólica post-9A. Adicionalmente, hay dos aspectos destacables para esta fase de término. Primero, que la superficie del cono aluvial en su zona proximal quedo preservada como un depósito residual (lag deposit) de materiales gruesos (e.g. cantos), aspecto que denota un evento erosivo de finos luego del peak del evento en esta parte del cono. Segundo, la presencia de fracturas de desecación en arcillas limosas en la superficie distal del cono denota la presencia de apozamientos en superficie y su posterior evaporación post evento 9A (Figura No 8B). Estas fracturas se desarrollaron en la capa superior del aluvio hasta tiempo después de terminado el evento $9 \mathrm{~A}$, dado que pozas de agua aún existían hasta al menos un mes después del 9 de agosto. Este contexto de depósito aluvional ha sido difícilmente constatado en estudios previos, principalmente aplicados en quebradas localizadas en sectores urbanizados en la planicie litoral y no en las cuencas sobre la cordillera de la Costa (Gran Escarpe costero) en el norte de Chile (Vargas et al., 2000; Grijalba, 2016).

Figura № 13

Esquema evolutivo del evento 9A en Alto Patache. El color azul son las quebradas de Alto Patache. Las flechas negras, la dirección de flujo. Las líneas segmentadas son regueros. El color anaranjado es el cono aluvial.

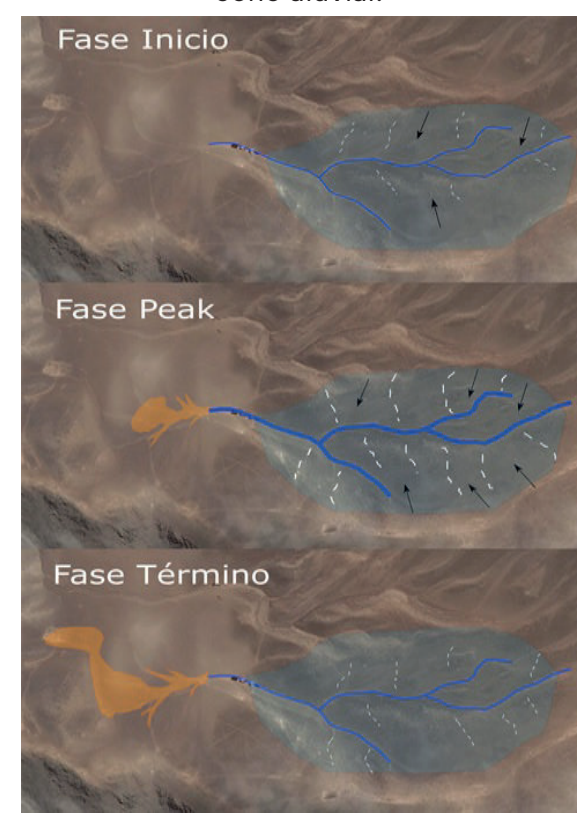

Fuente: Elaboración propia.

\section{Recurrencia de aluviones en el desierto y ENSO}

La secuencia estratigráfica tipo expuesta en las quebradas, se compone de una unidad basal (Unidad 1) que corresponde a un estrato carbonatado, a la cual sobreyacen brechas sedimentarias 
clasto soportado (Unidad 2), atribuido a eventos aluviales previo al 9A. Sobre este estrato, se encuentran depósitos aluviales (unidad 2), los cuales son más recientes que la unidad que subyace de la que se diferencia además por presentas raíces finas y conchas de Bostrix delerictus Broderip (Figura $N^{\circ} 10$ ). Respecto de las unidades aluvionales presentes en la estratigrafía, estas representan dos tipos de flujo recurrentes en el tiempo: detrítico y de barro (Hungr, 2005). Ambos tipos ocurrieron durante el evento del 9A también. El carácter fluvial y aluvial están bien representados por las facies en los sedimentos de la Figura No 8 del cono aluvial igualmente, lo que sugiere que la dinámica del desierto costero puede sufrir cambios geomorfológicos en periodos cortos de tiempo que favorecen mayores tasas de erosión, transporte y depositación aluvial (Orellana, 2010; Quezada et al., 2010). Flujos detríticos y de barro pre-9A fueron constatados en las columnas estratigráficas I y $\mathrm{P}$ (Figura $\mathrm{N}^{\circ} 10$ ). Aquí los sedimentos muestran un rango amplio desde facies arenosas que contienen clastos esparcidos hasta facies gravosas sin o con bajo contenido de matriz. A pesar de que ambas incluyen estructuras de flujo, se denota una diferencia en el contenido de agua del flujo que originó el sedimento: mientras las gravas clasto-soportadas indican un carácter fluvial (dominancia de agua), la mayor proporción de matriz (e.g. arena) denota un flujo aluvial relativamente más viscoso y cohesivo, en respuesta al mayor contenido de sedimento fino transportado (Hungr, 2005). Por otro lado, la estratigrafía también evidencia periodos de estabilidad geomorfológica que se intercalan con la actividad morfogenética de quebradas. En este sentido, los depósitos carbonatados parecen constituir paleosuelos enterrados donde primó la pedogénesis por sobre la morfogénesis (columna estratigráfica I). Periodos de esta índole sin actividad de quebradas han sido descritos para el Holoceno en el norte de Chile (Vargas et al., 2000; Vargas et al., 2006), denotando la dinámica de estabilidad del desierto interrumpida con cierta recurrencia por lluvias intensas de gran impacto en el paisaje.

El desarrollo de los eventos aluviales en la Cordillera de la Costa del Desierto de Atacama y sur del Perú se ha relacionado con fases cálidas del ENSO (fases El Niño) (Garreaud y Rutllant, 1996; Vargas et al., 2000; Vargas et al., 2006; Grijalba, 2016). Durante estos años El Niño se crean condiciones océano-atmosféricas para que borrascas irrumpan en el desierto aprovechando un Anticiclón del Pacifico debilitado y descargando lluvias en los cerros costeros (Garreaud y Rutllant, 1996). Por ejemplo, durante el año El Niño 2015 (fuerte) hubo crecidas súbitas de ríos y flujos de barro en varias cuencas del desierto de Atacama (23-25 de marzo: Antofagasta, Chañaral, Paipote; 9 agosto: Tocopilla, Alto Patache). Otro ejemplo es lo ocurrido durante el fenómeno El Niño del 2002, en donde la estación meteorológica de lquique midió $6 \mathrm{~mm}$ de precipitación para el 2 de julio de dicho año (Quezada, 2006). Situación similar ocurrió en Alto Patache en El Niño de 1997. Aunque las lluvias no fueron cuantificadas in situ, se observó una profundización de la humedad del suelo hasta c.30 cm de profundidad (Horacio Larraín, comunicación personal). Ese año no se desencadenó ningún flujo aluvional en Alto Patache, probablemente debido a un volumen e intensidad de agua por bajo el umbral necesario gatillar escorrentía superficial (Vargas et al., 2000; Haug et al., 2010). Por ejemplo, Haug et al., (2010) determinó que para producir procesos aluviales entre Iquique y Antofagasta se debe superar un umbral de $>4 \mathrm{~mm} /$ hora. Este umbral probablemente no fue superado en 1997 en Alto Patache, a diferencia del 9A, cuando fue ampliamente sobrepasado. No obstante, en 1997 en otras cuencas del desierto de Atacama si se desencadenaron crecidas hidrológicas y aluviones en respuesta a las lluvias de alta intensidad de (por ejemplo, Antofagasta, Copiapó). Es muy probable que los aluviones presentes en el registro estratigráfico se hallan desencadenado por lluvias anómalas en años El Niño, por ejemplo, durante el Holoceno (Vargas et al., 2000; Vargas et al., 2006), aunque aquellos registros previos del Pleistoceno pueden corresponder a tipos climáticos más húmedos que caracterizaron el último periodo glacial 
en el área (Ortlieb y Vargas 2003). Nuevos antecedentes geo-cronométricos de Alto Patache son necesarios para reconstruir su historia aluvial y sus causas.

\section{Conclusiones}

La lluvia del 9A registrada en la Estación Atacama UC - Oasis Niebla Alto Patache fue de un total de 51,6 mm en 6 horas. Este total de precipitación es completamente anómalo para la región, donde la precipitación normal anual es cercana a $0 \mathrm{~mm}$. La precipitación superó con creces los umbrales y desencadenó aluviones en todas las quebradas costeras de Alto Patache. En la salida de nuestra cuenca de control estimamos caudales medios del orden de $11 \mathrm{~m}^{3} \mathrm{~s}^{-1}$, los que erosionaron, transportaron y depositaron un total de al menos c.640 $\mathrm{m}^{3}$ de sedimentos aluviales. Tanto las quebradas como las laderas de los cerros aledaños fueron profundamente modificados por la erosión de la escorrentía.

La estratigrafía del depósito aluvial 9A concuerda directamente con la dinámica de las lluvias medidas ese día en Alto Patache, a partir de lo cual se propone un modelo esquemático con fases Inicio, Peak y Término que correlacionan las etapas progresivas de la vaguada con la depositación de sedimentos en el cono aluvial (Figura № 13).

El aluvión 9A descrito en este estudio tiene precedentes en Alto Patache. Se trata entonces de procesos morfodinámicos recurrentes en el tiempo, propios de la dinámica en la cordillera de la Costa del Atacama. Eventos como el 9A son gatillados por las precipitaciones anómalas favorecidas por las teleconexiones climáticas tropicales y extratropicales durante fases El Niño, que es parte de la climatología de la región (p. ej., del Río et al., en prensa). Este aspecto debe considerarse en la planificación de asentamientos dado que comprende una amenaza directa a la población localizada en lechos de quebradas secas del desierto de Atacama.

\section{Referencias bibliográficas}

BOGGS, S. Principles of sedimentology and stratigraphy. New Jersey: Editorial Pearson Prentice Hall, 2012.

CERECEDA, P.; LARRAIN, H.; OSSES, P.; FARÍAS, M. \& EGAÑA, I. The climate of the coast and fog zone in the Tarapacá Region, Atacama Desert, Chile. Atmospheric Research, 2008(a), No 87, p. 301-311.

CERECEDA, P.; LARRAIN, H.; OSSES, P.; FARÍAS, M \& EGAÑA, I. The spatial and temporal variability of fog and its relation to fog oases in the Atacama Desert, Chile. Atmospheric Research, 2008(b), No 87, p. 312-323.

DEL RÍO, C.; GARCIA, J.L.; OSSES, P.; ZANETTA, N.; LAMBERT, F.; RIVERA, D.; SIEGMUND, A.; WOLF, N.; CERECEDA, P.; LARRAIIN, H. \& LOBOS, F. ENSO Influence on Coastal Fog-Water Yield in the Atacama Desert, Chile. Aerosol and Air Quality Research, 2017 (en prensa). 
DUNAI, T.; GONZALEZ, G \& JUEZ, J. Oligocene-Miocene age of aridity in the Atacama Desert revealed by exposure dating of erosion-sensitive landforms. Geology, 2005, № 33, p. 321-324.

EGAÑA, I.; PINTO, R.; CERECEDA, P.; LARRAIN, H.; OSSES, P y FARIAS, M. Estudio biogeográfico de la comunidad arbustiva del farellón costero de Punta Patache, lquique, Chile. Revista de Geografía Norte Grande, 2004, № 31, p. 99-113.

GARREAUD, R. The climate of northern Chile: Mean state, variability and trends. "Astronomical Site Testing Data Conference in Chile". Revista Mexicana de Astronomía y Astrofísica (SC), 2011, No 41, p. 5-11.

GARREAUD, R.; BARICHIVICH, J.; CHRISTIE, D \& MALDONADO, A. Interannual variability of the coastal fog at Fray Jorge relicts' forests in semiarid Chile. Journal Geophysical Research, 2008, No 113, p. 1-16.

GARREAUD, R. y RUTLLANT, J. Análisis meteorológico de los aluviones de Antofagasta y Santiago de Chile en el periodo 1991-1993. Atmósfera, 1996, № 9, p. 251-271.

GRIJALBA, V. Geología y análisis histórico-meteorológico del aluvión de marzo de 2015 en Chañaral, Atacama. Santiago de Chile: Memoria para optar al Título de Geólogo, Departamento de Geología, Facultad de Ciencias Físicas y Matemáticas, Universidad de Chile, 2016.

HARTLEY, A.; CHONG, G.; HOUSTION, J. \& MATHER, A. 150 Million years of climatic stability: evidence from the Atacama Desert, northern Chile. Journal of the Geological Society, 2005, No 162, p. 421-424.

HAUG, E.; KRAAL, E.; SEWALL, J.; VAN DIJK, M. \& CHONG, G. Climatic and geomorphic interactions on alluvial fans in the Atacama Desert, Chile. Geomorphology, 2010, № 121, p. 184-196.

HAUSER, A. Remociones en masa en Chile. Santiago de Chile: Boletín Servicio Nacional de Geología y Minería, 2000.

HOUSTON, J. Variability of precipitation in the Atacama Desert: Its causes and hydrological impact. International Journal of Climatology, 2006, № 26, p. 2181-2198.

HUNGR, O. Classification and terminology. In: JAKOB, M. \& HUNGR, O. Debris flow hazard and related phenomena. Chichester: Editorial Springer-Praxis, 2005.

LATORRE, C.; GONZALEZ, A.L.; QUADE, J.; FARIÑA, J.M.; PINTO, R. \& MARQUET, P. Establishment and formation of fog-dependent Tillandsia landbeckii dunes in the Atacama Desert: Evidence from radiocarbon and stable isotopes. Journal of Geophysical Research, 2011, № 116, p. 1-12.

MANRIQUE, R.; FERRARI, C. \& PEZZI, G. The influence of El Niño Southern oscillation (ENSO) on fog oases along the Peruvian and Chilean coastal deserts. In: V International Conference on Fog, Fog Collection and Dew. Münster, Germany, 2010. 
MCGOWEN, J. \& GROAT, C. Van Horn Sandstone, West Texas, an alluvial fan model for mineral exploration. Texas Bureau of Economic Geology, Report of Investigations, 1971, No 72, p. 1-57.

MIALL, A. Stratigraphy: A modern synthesis. Switzerland: Editorial Springer, 2016.

NISHIIZUMI, K.; FINKEL, R.L.; BRIMHALL, G.; MOTE, T.; MUELLER, G. \& TIDY, E. Ancient exposure ages of alluvial fan surfaces compared with incised stream beds and bedrock in the Atacama Desert of North Chile. In: Geological Society of America Annual Meeting. Abstracts with Programs, 1998, Vol. 30, p. 298.

NATIONAL OCEANIC AND ATMOSPHERIC ADMINISTRATION. Southern Oscillation Index (SOI), 2017a. Disponible en internet: http://www.cpc.ncep.noaa.gov/data/indices/soi

NATIONAL OCEANIC AND ATMOSPHERIC ADMINISTRATION. Climate prediction center. Historical El Niño/La Niña episodes (1950-present), 2017b. Disponible en internet: http://www.cpc.noaa.gov/ products/analysis_monitoring/ensostuff/ensoyears.shtml

ORELLANA, H. Aspectos geodinámicos del desierto costero de Atacama, sector Alto Patache (oasis niebla) y bajo Patache. Santiago de Chile: Memoria para optar al Título de Geógrafo, Escuela de Geografía, Universidad de Chile. 2010.

ORTEGA, C. Variabilidad climática en la costa semiárida de Chile $\left(30^{\circ}-32^{\circ} \mathrm{S}\right)$ durante los últimos 13.000 años. Santiago de Chile: Tesis para optar al Grado de Doctora en Ciencias, mención Geología, Departamento de Geología, Facultad de Ciencias Físicas y Matemáticas, Universidad de Chile, 2014.

ORTLIEB, L. \& VARGAS, G. Debris-flow deposit and El Niño Impacts along the hyperarid southern Peru coast. In: HAAS, J. \& DILLON, M. El Niño in Peru: Biology and culture over 10,000 years. Chicago: Ediciones Fieldiana Botany, 2003, № 34, p. 24-51.

ORTLIEB, L. Eventos El Niño y episodios Iluviosos en el Desierto de Atacama: el registro de los dos últimos siglos. Bulletin de l'Institut Francais d'Etudes Andines, 1995, Vol. 24, No 3, p. 519-537.

PINTO, R.; LARRAÍN, H.; CERECEDA, P.; LÁZARO, P. \& OSSES, P. Monitoring fog vegetation at a fog site in Alto Patache South of Iquique, Chile during El Niño and La Niña events. In: Proceedings Second Conference on Fog and Fog collection, St. John's, Canada, julio de 2001.

QUEZADA, J.; CERDA, J.L. y JENSEN, A. Efectos de la tectónica y el clima en la configuración morfológica del relieve costero del norte de Chile. Andean Geology, 2010, Vol. 37, № 1, p. 78-109.

QUEZADA, J. Fenómenos climáticos anómalos en el litoral del norte de Chile y su impacto en el relieve. En: XI Congreso Geológico Chileno, Antofagasta, Chile, agosto de 2006.

SEPÚlVEDA, F.; VÁSQUEZ, P. y QUEZADA, A. Carta Patillos y Oficina Victoria, región de Tarapacá, Escala 1:100.000. Santiago de Chile: Carta Geológica de Chile, Serie Geología Básica, SERNAGEOMIN, 2014. 
VARGAS, G.; RUTLLANT, J \& ORTLIEB, L. ENSO tropical-extratropical climate teleconnections and mechanisms for Holocene debris flows along the hyperarid coast of western South America $\left(17^{\circ}\right.$. $\left.24^{\circ} \mathrm{S}\right)$. Earth and Planetary Science Letters, 2006, No 249, p. 467-483.

VARGAS, G.; ORTLIEB, L. y RUTLLANT, J. Aluviones históricos en Antofagasta y su relación con eventos El Niño/ Oscilación del Sur. Revista Geológica de Chile, 2000, Vol. 47, No 2, p. 157-176.

VARGAS, G. y ORTLIEB, L. Patrones de variaciones climáticas durante el Cuaternario tardío en la costa de la región de Antofagasta, Chile. Bulletin Institute francés Études Andine, 1998, Vol. 27, No 3, p. 385-394. 
